OSU-HEP-03-8

May 2003

\title{
Gauged Baryon Parity and Nucleon Stability
}

\author{
K.S. Babu ${ }^{1}$, Ilia Gogoladze ${ }^{2}$ and Kai Wang ${ }^{3}$ \\ Department of Physics, Oklahoma State University \\ Stillwater, OK 74078-3072, USA
}

\begin{abstract}
We show that the Standard Model Lagrangian, including small neutrino masses, has an anomaly-free discrete $Z_{6}$ symmetry. This symmetry can emerge naturally from $\left(I_{R}^{3}+L_{i}+L_{j}-2 L_{k}\right)$ gauge symmetry ( $L_{i}$ is the $i$ th lepton number) and ensure the stability of the nucleon even when the threshold of new physics $\Lambda$ is low. All $\Delta B=1$ and $\Delta B=2\left(B\right.$ is the baryon number) effective operators are forbidden by the $Z_{6}$ symmetry. $\Delta B=3$ operators are allowed, but they arise only at dimension 15 . We estimate the lifetime for "triple nucleon decay" resulting from these operators and find that $\Lambda$ can be as low as $10^{2} \mathrm{GeV}$. We suggest a simple mechanism for realizing reasonable neutrino masses and mixings even with such a low scale for $\Lambda$.
\end{abstract}

\footnotetext{
${ }^{1}$ E-mail address: babu@okstate.edu

${ }^{2}$ On leave of absence from: Andronikashvili Institute of Physics, GAS, 380077 Tbilisi, Georgia. E-mail address: ilia@hep.phy.okstate.edu

${ }^{3}$ E-mail address: wangkai@hep.phy.okstate.edu
} 


\section{Introduction}

The Standard Model (SM) has been highly successful in explaining all experimental observations in the energy regime up to a few hundred GeV. However, it is believed to be an effective field theory valid only up to a cutoff scale $\Lambda$. Non-renormalizable operators which are gauge invariant but suppressed by appropriate inverse powers of $\Lambda$ should then be considered in the low energy effective theory [1. The dimension 5 operator $\ell \ell H H / \Lambda_{L}(\ell$ is the lepton doublet) which violates lepton number $(L)$ by two units is the lowest dimensional of such operators. Experimental evidence for neutrino masses suggests the effective scale of $L$-violation is around $\Lambda_{L} \sim 10^{14}-10^{15} \mathrm{GeV}$. The $d=6$ operator $Q Q Q \ell / \Lambda_{B}^{2}$ violates both baryon number $(B)$ and lepton number and leads to the decay of the nucleon. The current limits on proton lifetime are $\tau_{p}>5 \times 10^{33}$ yrs for $p \rightarrow e^{+} \pi^{0}$ and $\tau_{p}>1.6 \times 10^{33}$ yrs for $p \rightarrow \bar{\nu} K^{+}$[2]. These limits imply that $\Lambda_{B}>10^{15} \mathrm{GeV}$. Grand Unified Theories with or without supersymmetry generate such $B$-violating operator with $\Lambda_{B} \sim 10^{14}-10^{16} \mathrm{GeV}$. These theories are currently being tested through nucleon decay. Any new physics with a threshold $\Lambda$ less than the GUT scale will thus be constrained by both proton lifetime and neutrino masses [1, 3, 4.

It is widely anticipated that new physics will show up around the $\mathrm{TeV}$ scale. This expectation is based on the stability of the Higgs mass. Supersymmetric theories with threshold of a few hundred GeV have been extensively studied as a solution to the Higgs mass problem (or the hierarchy problem). Indeed, these theories allow for both $B$ and $L$ violating operators. Care must be (and usually is) taken to satisfy the experimental constraints.

A second scenario that has been widely discussed recently to address the hierarchy problem is the possible existence of large extra dimensions [5]. Here the fundamental scale of gravity $M_{\mathrm{Pl}}$ is lowered from its 4 dimensional value of $10^{19} \mathrm{GeV}$ to $M_{*} \sim$ a few TeV. Nonrenormalizable operators associated with quantum gravity which are only suppressed by inverse power of $M_{*}$ will then be present in the low energy theory. $L$ and $B$ violation would put major constraints on these theories. Similar remarks will apply to other solutions to the hierarchy problem, such as composite Higgs, little Higgs [6], etc.

In this paper we address the role discrete gauge symmetries [7] can play in suppressing baryon number and lepton number violating processes even when the scale of new physics is low. The SM effective lagrangian does not have a continuous anomaly-free symmetry that can suppress these processes. This is our reason for focusing on discrete symmetries. It is preferable that such symmetries have a gauge origin since all global symmetries are expected to be violated by the quantum gravitational effects [8]. We find that the SM lagrangian, including small neutrino masses, has an anomaly-free $Z_{6}$ symmetry. This $Z_{6}$ acts as discrete baryon number and forbids potentially dangerous $\Delta B=1$ and $\Delta B=2$ nucleon decay processes. $\Delta B=3$ operators are allowed, but they arise only as $d=15$ operators. "Triple nucleon decay" can proceed through these operators; we estimate its rate and find $\Lambda_{B}$ can be as low as $10^{2} \mathrm{GeV}$. We show that the $Z_{6}$ symmetry has a natural gauge origin in the anomaly-free $U(1)$ corresponding to $I_{R}^{3}+L_{i}+L_{j}-2 L_{k}$, where $L_{i}$ is $i$ th lepton number and $I_{R}^{3}$ is the third component of the righthanded isospin. No new particles are introduced to cancel anomalies except the righthanded neutrinos. We show how an additional $Z_{5}$ or $Z_{7}$ 
gauge symmetry generates the desired neutrino masses and mixings even with a low scale for $\Lambda$.

Discrete gauge symmetries have been utilized in suppressing nucleon decay [9] as well as in addressing other aspects of physics such as solving the $\mu$ problem of supersymmetry, fermion mass hierarchy problem [10, 11] and the stability of the axion [11. A $Z_{3}$ baryon parity was found in Ref. 9] that suppresses nucleon decay. In order for it to have a gauge origin, extra particles were introduced. ${ }^{4}$ There have been also attempts to gauge baryon number by introducing a fourth family of fermions [12], or with the use of extra dimensions 13. The spirit of this paper is similar to that of Ref. [9, 12, 13, our models are however much more economical.

\section{Gauged Baryon Parity and Suppression of Proton Decay}

In this section we show that the SM lagrangian has a discrete $Z_{6}$ gauge symmetry which forbids all $\Delta B=1$ and $\Delta B=2$ baryon violating effective operators. This can be seen as follows. The SM Yukawa couplings incorporating the seesaw mechanism to generate small neutrino masses is

$$
\mathcal{L}_{\mathrm{Y}}=Q u^{c} H+Q d^{c} H^{*}+\ell e^{c} H^{*}+\ell \nu^{c} H+M_{R} \nu^{c} \nu^{c} .
$$

Here we have used the standard (lefthanded) notation for the fermion fields and have not displayed the Yukawa couplings or the generation indices. This lagrangian respects a $Z_{6}$ discrete symmetry with the charge assignment as shown in Table 1]. Also shown in Table [1 are the charge assignments under the $Z_{3}$ and $Z_{2}$ subgroups of $Z_{6}$. The $Z_{3}$ assignment is identical to that in Ref. 9]

\begin{tabular}{|l|ccccccc|}
\hline & $Q$ & $u^{c}$ & $d^{c}$ & $\ell$ & $e^{c}$ & $\nu^{c}$ & $H$ \\
\hline$Z_{6}$ & 6 & 5 & 1 & 2 & 5 & 3 & 1 \\
$Z_{3}$ & 3 & 2 & 1 & 2 & 2 & 3 & 1 \\
$Z_{2}$ & 2 & 1 & 1 & 2 & 1 & 1 & 1 \\
\hline
\end{tabular}

Table 1: Family-independent $Z_{6}$ charge assignment of the SM fermions and the Higgs boson along with the charges under $Z_{3}$ and $Z_{2}$ subgroups.

From Table 1 it is easy to calculate the $Z_{6}$ crossed anomaly coefficients with the SM gauge groups. We find the $S U(3)_{C}$ or $S U(2)_{L}$ anomalies to be

$$
\begin{aligned}
A_{\left[S U(3)_{C}\right]^{2} \times Z_{6}} & =3 N_{g} \\
A_{\left[S U(2)_{L}\right]^{2} \times Z_{6}} & =N_{g}
\end{aligned}
$$

\footnotetext{
${ }^{4}$ The charges of the SM particles under the $U(1)$ symmetry in Ref. [9] are $\{Q, u, d, l, e\}=$ $(0,-1,1,-1,-1)$. The model has extra leptons $l^{\prime}(3), \bar{l}^{\prime}(0), \bar{e}^{\prime}(3), \bar{e}^{\prime}(0)$ (where the $U(1)$ charges are given in parentheses) as well as exotic leptons with electric charge of $\pm 1 / 3$ and $0: 2 \times F^{1 / 3}(3), 1 \times F^{-1 / 3}(3)$, $2 \times F^{-1 / 3}(0), 1 \times F^{1 / 3}(0)$ and $5 \times N^{0}(-3), N^{0}(1), N^{0}(3)$.
} 
where $N_{g}$ is the number of generations. The condition for a $Z_{N}$ discrete group to be anomalyfree is

$$
A_{i}=\frac{N}{2} \bmod N
$$

where $i$ stands for $S U(3)_{C}$ and $S U(2)_{L}$. For $Z_{6}$, this condition reduces to $A_{i}=3 \bmod 6$, so when $N_{g}=3, Z_{6}$ is anomaly-free. Obviously, the $Z_{3}$ and $Z_{2}$ subgroups are also anomalyfree. The significance of this result is that unknown quantum gravitational effects will respect this $Z_{6}$. It is this feature that we utilize to stabilize the nucleon. Absence of anomalies also suggests that the $Z_{6}$ may have a simple gauge origin.

To see how the $Z_{6}$ forbids $\Delta B=1$ and $\Delta B=2$ processes, we note that it is a subgroup of $U(1)_{2 Y-B+3 L}$ where $Y$ is SM hypercharge [10. We list in Table 2 the charges under the three $U(1)$ symmetries. Comparing the last line of Table 2 with the charge assignment of Table 1 it is clear that the $Z_{6}$ is a subgroup of $U(1)_{2 Y-B+3 L}$. Any $Z_{6}$ invariant effective operator must then satisfy

$$
2 \Delta Y-\Delta B+3 \Delta L=0 \bmod 6 .
$$

Invariance under $U(1)_{Y}$ implies $\Delta Y=0$. Consider $\Delta B=1$ effective operators which must then obey (from Eq. (41) $3 \Delta L=1 \bmod 6$. This has no solution, since $3 \Delta L=0 \bmod 3$ from Table 2. Similarly, $\Delta B=2$ operators must obey $3 \Delta L=2 \bmod 6$ which also has no solution. $\Delta B=3$ operators, which corresponds to $3 \Delta L=0 \bmod 6$, are allowed by this $Z_{6}$. Such operators have dimension 15 or higher and have suppression factors of at least $\Lambda^{-11}$. These will lead to "triple nucleon decay" processes where three nucleons in a heavy nucleus undergo collective decays leading to processes such as $p n n \rightarrow e^{+} \pi^{0}$. We estimate the rates for such decay in Section 4 and find that $\Lambda$ can be as low as $10^{2} \mathrm{GeV}$.

\begin{tabular}{|c|ccccccc|}
\hline & $Q$ & $u^{c}$ & $d^{c}$ & $\ell$ & $e^{c}$ & $\nu^{c}$ & $H$ \\
\hline$U(1)_{B}$ & $1 / 3$ & $-1 / 3$ & $-1 / 3$ & 0 & 0 & 0 & 0 \\
$U(1)_{L}$ & 0 & 0 & 0 & 1 & -1 & -1 & 0 \\
$U(1)_{Y}$ & $1 / 6$ & $-2 / 3$ & $1 / 3$ & $-1 / 2$ & 1 & 0 & $1 / 2$ \\
$U(1)_{2 Y-B+3 L}$ & 0 & -1 & 1 & 2 & -1 & -3 & 1 \\
\hline
\end{tabular}

Table 2: Charge assignment under $B, L, Y$ and $U(1)_{2 Y-B+3 L}$ which contains the $Z_{6}$ of Table 1

\section{$3 \quad$ Embedding $Z_{6}$ in $I_{R}^{3}+L_{i}+L_{j}-2 L_{k}$}

It is interesting to see if the $Z_{6}$ symmetry of Table 1 can be realized as an unbroken subgroup of a gauged $U(1)$ symmetry. Although the $Z_{6}$ is a subgroup of the $U(1)_{(2 Y-B+3 L)}$, this $U(1)$ would be anomalous without enlarging the particle content. We have found a simple and economic embedding of $Z_{6}$ into a $U(1)$ gauge symmetry associated with $I_{R}^{3}+L_{i}+L_{j}-2 L_{k}$. Here $L_{i}$ is the $i$ th family lepton number and $i \neq j \neq k$. No new particles are needed to 
cancel gauge anomalies. With the inclusion of righthanded neutrinos $I_{R}^{3}=Y-(B-L) / 2$ is an anomaly-free symmetry. $L_{i}+L_{j}-2 L_{k}$, which corresponds to the $\lambda_{8}$ generator acting in the leptonic $S U(3)$ family space, is also anomaly-free.

The charges of the SM particles under this $U(1)$ are

$$
\begin{aligned}
& Q_{i}=(0,0,0), \quad u_{i}{ }^{c}=(-1,-1,-1), \quad d_{i}{ }^{c}=(1,1,1), \\
& \ell_{i}=(-4,2,2), \quad e_{i}{ }^{c}=(5,-1,-1), \quad \nu_{i}{ }^{c}=(3,-3,-3), \quad H=1 .
\end{aligned}
$$

This charge assignment allows all quark masses and mixings as well as charged lepton masses. When the $U(1)$ symmetry breaks spontaneously down to $Z_{6}$ by the vacuum expectation value of a SM singlet scalar field $\phi$ with a charge of 6 , realistic neutrino masses and mixings are also induced. The relevant lagrangian for the righthanded neutrino Majorana masses is

$$
\mathcal{L}_{\nu^{\mathrm{c}}}=M_{12} \nu_{1}^{c} \nu_{2}^{c}+M_{13} \nu_{1}^{c} \nu_{3}^{c}+\nu_{3}^{c} \nu_{3}^{c} \phi+\nu_{2}^{c} \nu_{2}^{c} \phi+\nu_{1}^{c} \nu_{1}^{c} \phi^{*}+\nu_{3}^{c} \nu_{2}^{c} \phi
$$

After integrating out the heavy righthanded neutrinos we obtain the following $\Delta L=2$ effective operators:

$$
\mathcal{L}_{\Delta L=2}=\frac{1}{\Lambda}\left(\ell_{1} \ell_{2} H H+\ell_{1} \ell_{3} H H+\ell_{1} \ell_{1} H H \epsilon+\ell_{2} \ell_{2} H H \epsilon^{*}+\ell_{2} \ell_{3} H H \epsilon^{*}+\ell_{3} \ell_{3} H H \epsilon^{*}\right) .
$$

Here $\Lambda \sim M_{12} \sim M_{13}$ is the scale of $L$-violation and we have defined $\epsilon \equiv\langle\phi\rangle / \Lambda$. For $\epsilon \ll 1$, this lagrangian leads to the inverted mass hierarchy pattern for the neutrinos which is well consistent with the current neutrino oscillation data. This neutrino mass mixing pattern is analogous to the one obtained from $L_{e}-L_{\mu}-L_{\tau}$ symmetry [14. However, here the $U(1)$ is a true gauge symmetry.

We have also investigated other possible $U(1)$ origin of the $Z_{6}$ symmetry and found the $I_{R}^{3}+L_{i}+L_{j}-2 L_{k}$ combination to be essentially unique. To see this, let us assign a general $U(1)_{X}$ charge for $i$ th generation of the SM fermions consistent with the $Z_{6}$ symmetry as

$$
\left\{Q_{i}, u_{i}^{c}, d_{i}^{c}, \ell_{i}, e_{i}^{c}, \nu_{i}^{c}\right\}=\left\{6 m_{1}^{(i)}, 5+6 m_{4}^{(i)}, 1+6 m_{3}^{(i)}, 2+6 m_{2}^{(i)}, 5+6 m_{5}^{(i)}, 3+6 m_{6}^{(i)}\right\}
$$

where $m_{j}^{(i)}$ are all integers. The Higgs field has a charge $H=1+6 m_{0}$. If we impose the invariance of the Yukawa couplings of the charged fermions and Dirac neutrinos for each generation, the anomaly coefficients from $i$ th generation become

$$
\begin{aligned}
A_{\left[S U(3)_{C}\right]^{2} \times U(1)_{X}}^{(i)} & =0 \\
A_{\left[S U(2)_{L}\right]^{2} \times U(1)_{X}}^{(i)} & =1+9 m_{1}^{(i)}+3 m_{2}^{(i)} \\
A_{\left[U(1)_{Y}\right]^{2} \times U(1)_{X}}^{(i)} & =-\left(1+9 m_{1}^{(i)}+3 m_{2}^{(i)}\right) \\
A_{\left[U(1)_{X}\right]^{2} \times U(1)_{Y}}^{(i)} & =\left[5+m_{0}\right] A_{\left[S U(2)_{L}\right]^{2} \times U(1)_{X}}^{(i)} \\
A_{\left[U(1)_{X}\right]^{3}}^{(i)} & =\left[5+m_{0}\right]^{2} A_{\left[S U(2)_{L}\right]^{2} \times U(1)_{X}}^{(i)} .
\end{aligned}
$$

The coefficient for the mixed gravitational anomaly for each generation is zero. From Eq. (17), it follows that $A_{2}=\sum_{i} A_{\left[S U(2)_{L}\right]^{2} \times U(1)_{X}}^{(i)}=\sum_{i}\left(1+9 m_{1}^{(i)}+3 m_{2}^{(i)}\right)=0$ can be satisfied 
only when all three generation contributions are included. Once $A_{2}=0$ is satisfied, all other anomaly coefficients will automatically vanish. $A_{2}=0$ can be rewritten in a familiar form as $3 \sum_{i} Q_{i}+\sum_{i} \ell_{i}=0$. Thus we see that any $U(1)$ symmetry satisfying this condition and consistent with the $Z_{6}$ charge assignment can be a possible source of $Z_{6}$. If the $Q_{i}$ are different for different generations, quark mixings cannot be generated without additional particles. By making a shift proportional to hypercharge, we can set $Q_{i}=0$ for all $i$. Two obvious solutions to $\sum_{i} \ell_{i}=0$ are $\ell_{i}=(1,1,-2)$ and $\ell_{i}=(1,-1,0)$. The latter one does not reproduce the $Z_{6}$ charge assignment while the former one does, which is our solution when $I_{R}^{3}$ is added to it.

A related $B-3 L_{\tau}$ has been discussed in Ref. [15]. This is the same as $B-L$ plus $L_{e}+L_{\mu}-2 L_{\tau}$. In Ref [15], only one righthanded neutrino $\nu_{\tau}^{c}$ is introduced so the seesaw mechanism applies only for one light neutrino. The other two neutrinos receive small masses from radiative correction. In our model, since there are three righthanded neutrinos, all the neutrino masses arise from the conventional seesaw mechanism.

\section{$4 \Delta B=3$ Operators and Triple Nucleon Decay}

We now list the lowest dimensional $(\mathrm{d}=15) \Delta B=3$ effective operators which are consistent with the $Z_{6}$ symmetry. Imposing gauge invariance and Lorentz invariance, we find them to be:

$$
\begin{aligned}
& \bar{u}^{c^{4}} \bar{d}^{5} \bar{e}^{c}, \quad \bar{u}^{c^{2}} \bar{d}^{c^{7}} e^{c}, \quad Q \bar{u}^{c^{3}} \bar{d}^{c^{5}} \ell, \quad Q \bar{u}^{c^{2}} \bar{d}^{6} \bar{\ell}, \quad Q^{2} \bar{u}^{c^{3}} \bar{d}^{c^{4}} \bar{e}^{c}, \\
& Q^{2} \bar{u}^{c} \bar{d}^{6} e^{c}, \quad Q^{3} \bar{u}^{c^{2}} \bar{d}^{4} \ell, \quad Q^{3} \bar{u}^{c} \bar{d}^{c^{5}} \bar{\ell}, \quad Q^{4} \bar{u}^{c^{2}} \bar{d}^{c^{3}} \bar{e}^{c}, \quad Q^{4} \bar{u}^{c} \bar{d}^{c^{4}} \nu^{c}, \\
& Q^{4} \bar{d}^{c^{5}} e^{c}, \quad Q^{5} \bar{u}^{c} \bar{d}^{c^{3}} \ell, \quad Q^{5} \bar{d}^{4} \bar{\ell}, \quad Q^{6} \bar{u}^{c} \bar{d}^{c^{2}} \bar{e}^{c}, \quad Q^{7} \bar{d}^{2} \ell, \quad Q^{8} \bar{d}^{c} \bar{e}^{c} .
\end{aligned}
$$

Here Lorentz, gauge and flavor indices are suppressed. These operators can lead to "triple nucleon decay". The dominant processes are

$$
\begin{aligned}
& \text { ppp } \rightarrow e^{+}+\pi^{+}+\pi^{+} \\
& p p n \rightarrow e^{+}+\pi^{+} \\
& p n n \rightarrow e^{+}+\pi^{0} \\
& n n n \rightarrow \bar{\nu}+\pi^{0} .
\end{aligned}
$$

Tritium $\left({ }^{3} \mathrm{H}\right)$ and Helium-3 $\left({ }^{3} \mathrm{He}\right)$ are examples of three-nucleon systems in nature. These nuclei are unstable and undergo $\beta$-decay with relatively short lifetime. In the presence of operators of Eq. (8),${ }^{3} H \rightarrow e^{+}+\pi^{0}$ and ${ }^{3} H e \rightarrow e^{+}+\pi^{+}$decays can occur. However, there is no stringent experimental limits arising from these nuclei. So we focus on triple-nucleon decay in Oxygen nucleus where there are experimental constraints from water detectors. To estimate the decay lifetime we need to first convert the nine-quark operators of Eq. (8) into three-nucleon operators and subsequently into Oxygen nucleus.

We choose a specific operator $Q^{5} \bar{d}^{c^{4}} \bar{\ell} / \Lambda^{11}$ as an example to study the process $p n n \rightarrow$

$e^{+}+\pi^{0}$ triple nucleon decay process. This induces the effective three-nucleon operator in Oxygen nucleus

$$
\frac{Q^{5} \bar{d}^{4} \bar{\ell}}{\Lambda^{11}} \sim \frac{\beta^{3}(1+D+F)}{\sqrt{2} f_{\pi} \Lambda^{11}}(\text { innpe })
$$


where $\beta \simeq 0.014 \mathrm{GeV}^{3}$ is the matrix element to convert three quarks into a nucleon [16]. $F \simeq 0.47, D \simeq 0.80$ are chiral lagrangian factors, and $f_{\pi}=139 \mathrm{MeV}$ is the pion decay constant.

We now estimate the wave-function overlap factor for three nucleons inside Oxygen nucleus to find each other. This is based on a crude free fermi gas model where the nucleons are treated as free particles inside an infinite potential well. A single nucleon wave function is given by $\psi_{m}(x)=\sqrt{2 / r} \sin (m \pi x / r)$, where $r$ is the size of nucleus and $m$ is the energy level. Incorporating isospin and Pauli exclusion principle, the highest energy level which corresponds to $m=4$ is found to have 2 protons and 2 neutrons. We assume the highest level has the most probability to form a Tritium-like "bound state" of three nucleons. The probability for three nucleons in Oxygen nucleus to overlap in a range the size of Tritium nucleus is

$$
P \sim \frac{4}{3} \int_{0}^{\sqrt[3]{\frac{3}{16}}} d\left(\frac{x_{1}}{r}\right) d\left(\frac{x_{2}}{r}\right) d\left(\frac{x_{3}}{r}\right)\left(\sin \left(\frac{4 \pi x_{1}}{r}\right) \sin \left(\frac{4 \pi x_{2}}{r}\right) \sin \left(\frac{4 \pi x_{3}}{r}\right)\right)^{2} \sim 0.0253,
$$

where $\sqrt[3]{\frac{3}{16}}$ is the ratio between the radii of Tritium nucleus and Oxygen nucleus, since $R \propto A^{1 / 3}$ ( $\mathrm{A}$ is the atomic number). So the effective baryon number violating operator of Eq. (10) becomes

$$
\frac{P \beta^{3}}{\sqrt{2} f_{\pi} \Lambda^{11} R^{3}}\left({ }^{3} H \pi e\right) .
$$

The triple nucleon decay lifetime can then be estimated to be

$$
\tau \sim \frac{16 \pi f_{\pi}^{2} \Lambda^{22} R^{6}}{P^{2} \beta^{6} M^{3} H}
$$

By putting the current limit on proton lifetime of $3 \times 10^{33}$ yrs, we obtain:

$$
\Lambda \sim 10^{2} \mathrm{GeV}
$$

Thus we see the $Z_{6}$ symmetry ensures the stability of the nucleon.

To test our crude model of nuclear transition, we have also evaluated the double nucleon decay rate within the same approach and found our results to be consistent with other more detailed evaluations [17].

\section{$5 \quad$ Realistic Neutrino Masses with Low Threshold}

If the threshold of new physics is low, but is still much larger than a TeV, small neutrino masses can be generated by the seesaw mechanism as discussed in Section 3 through the effective operators $\ell \ell H H / \Lambda$. If the threshold is as low as a few $\mathrm{TeV}$, the induced neutrino mass will be too large. Here we show a mechanism by which such operators can be suppressed by making use of a discrete $Z_{N}$ symmetry (with $N$ odd) surviving to low scale. This $Z_{N}$ has a natural embedding in $B-L$ gauge symmetry. 
Consider the following effective operators in the low energy lagrangian:

$$
\mathcal{L} \supset \ell \ell H H \frac{S^{6}}{\Lambda^{7}}+\frac{S^{2 N}}{\Lambda^{2 N-4}} .
$$

Here $S$ is a singlet field which has charge $(1,3)$ under $Z_{N} \times Z_{6}$ while $\ell$ has charge $(-3,2)$. (The $Z_{6}$ charges of SM particles are as listed in Table 1) The first term in Eq. (15) respects a $U(1)$ symmetry while the second term reduces this to $Z_{6} \times Z_{N}$. If $S$ develops a VEV of order $10^{2} \mathrm{GeV}$, realistic neutrino mass can arise even when $\Lambda$ is low. For example, if $\Lambda=10 \mathrm{TeV}$ and $S=10^{2} \mathrm{GeV}$, the neutrino mass is of order $v^{2}\langle S\rangle^{6} / \Lambda^{7} \sim 0.4 \mathrm{eV}$, which is consistent with the mass scale suggested by the atmospheric neutrino oscillation data.

Two explicit examples of the $Z_{N}$ symmetry with $N=5$ and 7 are shown in Table 5 . These $Z_{N}$ symmetries are free from gauge anomalies. In the $Z_{5}$ example, the crossed anomaly coefficients for $S U(3)_{C}$ and $S U(2)_{L}$ are $5 N_{g}$ and $5 N_{g} / 2$ respectively showing that $Z_{5}$ is indeed anomaly-free. For $Z_{7}$, these coefficients are $7 N_{g}$ and $7 N_{g} / 2$, so it is also anomaly-free.

\begin{tabular}{|c|ccccccc|}
\hline Field & $Q$ & $u^{c}$ & $d^{c}$ & $\ell$ & $e^{c}$ & $H$ & $S$ \\
\hline$Z_{5}$ & 1 & 4 & 4 & 2 & 3 & 0 & 1 \\
$Z_{7}$ & 1 & 6 & 6 & 4 & 3 & 0 & 1 \\
\hline
\end{tabular}

Table 3: $Z_{N}$ charge assignment for $N=5$ and 7 .

It is interesting to ask if the $Z_{N}$ can be embedded into a gauged $U(1)$ symmetry. A simple possibility we have found is to embed this $Z_{N}$ into the anomalous $U(1)_{A}$ symmetry of string origin with the anomalies cancelled by the Green-Schwarz mechanism [18]. Consider $U(1)_{B-L}$ without the right handed neutrinos but with the inclusion of vector-like fermions which have the quantum numbers of $\mathbf{5}(3)$ and $\overline{\mathbf{5}}(2)$ under $S U(5) \times U(1)_{A}$. This $U(1)_{A}$ is anomaly-free by virtue of the Green-Schwarz mechanism. When this $U(1)_{A}$ breaks down to $Z_{5}$, the extra particles get heavy mass and are removed from the low energy theory which is the $Z_{6} \times Z_{5}$ model.

Without the second term in Eq. (15), the phase of $S$ field will be massless upon spontaneous symmetry breaking. This Majoron field [19] would however acquire a mass from the second term of Eq. (15). In the $Z_{6} \times Z_{5}$ model, the mass of the Majoron is of order $\langle S\rangle^{7} / \Lambda^{6} \sim 100 \mathrm{keV}$. In the $Z_{6} \times Z_{7}$ model, the Majoron mass is of order $\langle S\rangle^{11} / \Lambda^{10} \sim 10 \mathrm{eV}$. Such a Majoron with a mass of either $100 \mathrm{keV}$ or $10 \mathrm{eV}$ is fully consistent with constraints from early universe cosmology [20. The interaction term $\ell \ell H H S^{6} / \Lambda^{7}$ induces the Majoron decay $S \rightarrow \nu \nu$ with a Yukawa coupling $Y_{S \rightarrow \nu \nu}=6 m_{\nu} /\langle S\rangle \sim 10^{-11}$. The decay rate of the Majoron can be estimated to be

$$
\Gamma=\frac{Y_{S \rightarrow \nu \nu}^{2} m_{S}}{8 \pi} \sim 10^{-23} m_{S}
$$

This corresponds to a Majoron lifetime of $\tau \sim 10$ sec for the $Z_{6} \times Z_{5}$ model and $\tau \sim$ $10^{5}$ sec for the $Z_{6} \times Z_{7}$ model. Such a Majoron can modify the big-bang nucleosynthesis processes. However the modification is not significant since the Majoron will decouple before 
the electro-weak phase transition. Its contribution to the expansion rate is equivalent to that of $0.047 \times 4 / 7 \sim 0.027$ light neutrino species [21]. This extra contribution is well within observational uncertainties.

\section{Conclusions}

We have shown in this paper that the SM lagrangian including small neutrino masses and with three generations has an anomaly-free $Z_{6}$ discrete symmetry. This $Z_{6}$ can act as gauged baryon parity and stabilize the nucleon even when the new physics threshold is below the TeV scale. The $Z_{6}$ has a natural embedding in $I_{R}^{3}+L_{i}+L_{j}-2 L_{k}$ gauge symmetry. We have also shown that realistic neutrino masses can be obtained through an additional anomaly-free $Z_{5}$ or $Z_{7}$ symmetry which may have its origin in the anomalous $U(1)_{A}$ symmetry.

Our framework can be readily extended to incorporate supersymmetry. The $Z_{6}$ charge assignment is still as in Table 11. The down-type Higgs $\bar{H}$ has a $Z_{6}$ charge of 5 and the Grassmann variable $\theta$ has charge 3 or $0\left[10\right.$. The Higgsino contribution to the $\left[S U(2)_{L}\right]^{2} \times Z_{6}$ anomaly is 3 and the gaugino contribution to $\left[S U(2)_{L}\right]^{2} \times Z_{6}$ and $\left[S U(3)_{C}\right]^{2} \times Z_{6}$ anomalies are 6 and 9 when $\theta$ charge is not zero. Since these contributions are all multiples of $6 / 2$, the $Z_{6}$ symmetry remains anomaly-free in supersymmetric case. This $Z_{6}$ forbids all $\Delta B=1$ and $\Delta B=2$ nucleon decay as in the non-supersymmetric case.

\section{Acknowledgement}

We are grateful to Yukihiro Mimura for useful discussion. This work is supported in part by DOE Grant \# DE-FG03-98ER-41076, a grant from the Research Corporation and by DOE Grant \# DE-FG02-01ER-45684.

\section{References}

[1] S. Weinberg, Phys. Rev. Lett. 43 (1979) 1566; F. Wilczek and A. Zee, Phys. Rev. Lett. 43 (1979) 1571.

[2] K. Hagiwara et al. [Particle Data Group Collaboration], Phys. Rev. D 66 (2002) 010001.

[3] H.A. Weldon and A. Zee, Nucl. Phys.B 173 (1980) 269.

[4] K.S. Babu and C.N. Leung, Nucl. Phys. B 619 (2001) 667.

[5] N. Arkani-Hamed, S. Dimopoulos and G. Dvali, Phys. Lett. B 429 (1998) 263; Phys. Rev. D 59 (1999) 086004; I. Antoniadis, N. Arkani-Hamed, S. Dimopoulos and G. Dvali, Phys. Lett. B 436 (1998) 257.

[6] N. Arkani-Hamed, A. G. Cohen, H. Georgi, Phys. Lett. B 513 (2001) 232.

[7] L. Krauss and F. Wilczek, Phys. Rev. Lett.182 (1989) 1221; T. Banks and M. Dine, Phys. Rev. D 45 (1992) 1424. 
[8] S.W. Hawking, Phys. Lett. B 195 (1987)337; G.V. Lavrelashvili, V.A. Rubakov and P.G. Tinyakov, JETP Lett. 46 (1987) 167; S. Giddings and A. Strominger, Nucl. Phys. B 306 (1988) 349; Nucl. Phys. B 321 (1989) 481; L.F. Abbot and M. Wise, Nucl. Phys. B 325 (1989) 687; S. Coleman and K. Lee, Nucl. Phys. B 329 (1989) 389; R. Kallosh, A. Linde, D. Linde and L. Susskind, Phys. Rev. D 52 (1995) 912.

[9] L.E. Ibáñez and G.G. Ross, Nucl. Phys. B 368 (1992) 3.

[10] K.S. Babu, I. Gogoladze and K. Wang, Nucl. Phys. B 660(2003) 322.

[11] K.S. Babu, I. Gogoladze and K. Wang, Phys. Lett. B 560 (2003) 214; K. Dimopoulos, et al., hep-ph/0303154.

[12] C.D. Carone and H. Murayama, Phys. Rev. D 52(1995) 484.

[13] T. Appelquist, et al. Phys. Rev. Lett. 87 (2001) 181802;C. Lee, Q. Shafi and Z. Tavartkiladze Phys. Rev. D 66 (2002) 055010; R.N. Mohapatra and A. Pérez-Lorenzana, Phys. Rev. D 67 (2003) 075015.

[14] See for example: K.S. Babu and R.N. Mohapatra, Phys. Lett. B 532 (2002) 77.

[15] E. Ma, Phys. Lett. B 433 (1998) 74.

[16] S. Aoki, et al. (JLQCD Collaboration) Phys. Rev. D 62 (2000) 014506.

[17] G. Feinberg, M. Goldhaber and G. Steigman, Phys. Rev. D 18 (1978) 1602; R. Mohapatra and G. Senjanović, Phys. Rev. Lett. 49 (1982) 7; L. Arnellos and W. J. Marciano, Phys. Rev. Lett. 48 (1982) 21; J. Basecq and L. Wolfenstein, Nucl. Phys. B 224 (1983) 21; S.P. Misra and U. Sarkar, Phys. Rev. D 28 (1983) 249; W.M. Alberico, et al, Phys. Rev. C 32 (1985) 1722; ; K. Benakli and S. Davidson, Phys. Rev. D 60 (1999) 025004; C.E. Carlson and C.D. Carone, Phys. Lett. B 512 (2001) 121.

[18] M.B. Green and J.H. Schwarz, Phys. Lett. B149 (1984) 117; Nucl. Phys. B255 (1985), 93; M. Green, J. Schwarz and P. West, ibid. B254 (1985) 327.

[19] Y. Chikashige, R.N. Mohapatra and R.D. Peccei, Phys. Lett. B 98 (1981) 265.

[20] I.Z. Rothstein, K.S. Babu and D. Seckel, Nucl. Phys. B 403 (1993) 725.

[21] See for example: The Early Universe, E.W. Kolb and M.S. Turner, Addison-Wesley Publishing Company, (1990) 